\title{
Role of stem cells in oral cancer
}

\section{Mini review}

Oral squamous cell carcinoma (OSCC) one of the top ten and widely occurring oral malignancy that lines the oral cavity. It arises in the squamous epithelium and it can be located or found on lips, gingiva, tongue, palate, buccal mucosa or floor of the mouth. ${ }^{1-3}$ Cancer stem cells (CSCs) research in the field of medicine and dentistry has been increased tremendously over the past few years. Despite recent progress in CSC research, knowledge of rare populations is still limited. Certain types of cancers are known to be multi-stage diseases, which generally progress into more malignant forms with the sequential accumulation of genetic and molecular alterations. ${ }^{4-7}$ For example, haematological malignancies, such as CML, are often found to have two distinct phases: chronic phase and blast crisis (or leukemia). Nevertheless, the misconception regarding CSCs and stem cells (SCs) remains constant. As the normal tooth derived SCs are considered same as oral CSCs. ${ }^{8-10}$ Therefore, the clarification of significant concepts relevant to normal SCs and CSCs is crucial along with the relevance of CSCs development in oral cancer is an essential need of an hour.

Stem cells are undifferentiated cells that have the ability to perpetuate themselves through self-renewal, repairing of damaged tissue and to generate mature cells of a particular tissue through differentiation. In humans, there are broadly two types of stem cells embryonic stem cells, which are derived from the inner cell mass of the blastocyst, are omnipotent and maintain their telomerase length thus capable of unlimited self-renewal. The second one is adult stem cells which are tissue specific have less capacity for self-renewal and ability to maintain their telomeres is limited and not sufficient to prevent their senescence therefore are at high risk of malignant transformation. Because over expression of telomerase activity in normal mesenchymal stem cells is correlated with accumulation of mutations. 4,5

Cancer stem cells (CSCs) also known as tumour-initiating cells possess similar characteristics to normal stem cells specifically having the ability of self-renewal and proliferation into multiple cell types, and express typical markers of stem cells. ${ }^{7}$ Such cells are resistant to drugs persist in tumours as a distinct population and cause relapse and metastasis by giving rise to new tumours. Genetic and molecular alterations and distinct pathologic abnormalities associated with different stages of cancer progression, it could be said that multiple CSC populations, either intrinsically linked or generated independently, responsible for different stages of cancer progression. The molecular mechanisms for the CSC formation and maintenance, their self-renewal regulation, which holds the key for the development of effective therapeutic strategies against CSC. Studies of stem cell biology are leading insight in the origins of cancer and will ultimately yield new approaches to fight this disease. ${ }^{11-13}$

\section{Cancer stem cell hypothesis and its implications}

If CSCs are relatively refractory, to treatment that have been given to eradicate the continuously dividing cells in tumour then they are unlikely to be curative and recurrence could occur. Since, the origin of CSCs remains unclear there had many hypothesis which had been proposed. If CSC hypothesis is correct then the concern would be to focus on the stem cell population instead of eliminating the bulk of rapidly dividing cells in tumours. CSC hypothesis is of utmost importance because it plays a vital role in changing basic cancer
Volume 10 Issue 5 - 2019

\author{
Rabia Sannam Khan,' Abdullah Chandra Talari \\ 'Department of Bio-Engineering, Lancaster University, UK \\ ${ }^{2}$ Experimental Officer in Bioengineering: Biomaterials and Tissue \\ Engineering, Lancaster University, UK
}

Correspondence: Rabia Sannam Khan, Department of BioEngineering, Lancaster University, Lancaster, LAI 4YW, UK, Email r.s.khan@lancaster.ac.uk

Received: September 03, 2019 | Published: October 04, 2019

researchers, clinical investigators, physicians, and cancer patients view cancer. ${ }^{14}$

\section{Old hypothesis}

\section{Cancer cells arise from primitive stem cells}

It was assumed that mutations responsible for transformation and progression occur in primitive cells only. Predicts little variability in the phenotype of the leukemic stem cells among different patients. ${ }^{15}$ Bonnet and Dick et al. ${ }^{9}$ performed experiments on normal and leukemic stem cells, which have the ability to repopulate immunedeficient mice with multiple lineages of normal and leukemic cells. ${ }^{8}$ For AML the disease in mice seen was initiated by primitive cell and they identified SCID leukemia initiating cell (SL-IC). ${ }^{16}$ They were not able to prove that SL-IC was actually leukemic stem cells, which had the potential for self-renewal. To determine if SL-IC from myelomonocytic samples had similar phenotype or to test the models explaining the origin and heterogeneity of AML because they were unable to perform secondary transplants or to transplant low cell doses or purified cells from myelomonocytic subtypes of AML using SCID recipients. The phenotype of SL-IC was similar to the normal stem cells regardless of the lineage markers expressed by the leukemic blasts or of the AML cell subtypes, suggesting that primitive normal stem cells rather than the committed progenitors are the target of leukemic transformation. ${ }^{17}$

\section{New hypothesis}

\section{Cancer cells arise from the progenitor cells}

As leukemogenic process causes increase in abnormal cells that are blocked at particular stage of differentiation the degree of target cell influences the characteristics of the resulting leukemic blasts. Only small population of leukemic cells maintain pool of non-proliferating blasts, it predicts that the phenotype of the leukemic stem cells from patients with myelomonocytic blasts, will differ from the leukemic stem cells from patients whose blasts expressed few lineage markers. ${ }^{8}$ Taussig et al. ${ }^{18}$ work suggested that leukemic progenitors acquire selfrenewal capacity and became second generation of SL-IC as the first generation comprised of SL-IC derived from HSC. So as the leukemia evolved Barbe et al reported that second-generation SL-ICs with a progenitor phenotype (CD34-CD38-) predominate and SL-ICs with a primitive phenotype (CD34-CD38-) were no longer detectable. ${ }^{18}$ 


\section{Oral cancer stem cells}

Stem cells are known to be responsible for body's normal tissue renewal and growth therefore it is logical to expect stem cells to be responsible for growth of oral cancer. Several evidences to reveal the CSCs major role in progression and pathogenesis of OSCC have been reported. The early studies of tissue culture suggested that oral cancer might have some stem cells because merely a sub population of oral cancer are capable of forming expanding colonies of tumours as well as responds to tumour initiating cells. CSCs isolation from oral cancers has been performed with CD44 marker. CD44 an adhesion molecule which had been identified previously in breast, prostate, pancreas and colon cancers. As for markers, no single marker is indicative of recognizing SCs. Therefore, Aldehyde dehydrogenase $(\mathrm{ALDH})$, which is an intracellular enzyme, improves the isolation and recognition of CSCs. Other markers suggested in several studies were CD133, c Met, phosphorylated STAT3, CK19, Oct-4, Sox2 \& Nanog, Klf4, Bmi. ${ }^{19,20}$ Substantial efforts to characterize and identify CSCs markers have been carried out in numerous types of tissues. Hence, a steady increase in the number of such markers can be identified. The research work on oral cancers and stem cells and markers had been carried out in smoker related Non-Asian populations and Asian population where the tobacco chewing is the major etiological factor is still to be explored. This could be attained by the use of methylome and miRNA profiles in oral cancer, microarray platforms and Next Generation Sequencing. ${ }^{21}$

\section{Conclusion}

Cancer stem cells are considered discrete populations of tumourresident stem cells that are responsible for tumour growth and progression. Targeting their elimination is expected to be a highly effective cancer therapy therefore the interest in identifying and characterizing these populations in human cance ${ }^{22}$ Normal stem cells are important for advance CSC research and critical pathways controlling stem cell properties. For this, identification of cell surface molecules for prospective stem cell isolation and biologically relevant stem cell assays are essential. In addition, technical improvement will expedite the studies of this rare and heterogeneous population. Further improvements in understanding of CSC, will be able to develop better diagnostic and therapeutic methodologies, with which to classify, treat, and cure cancer.

\section{Funding details}

None.

\section{Acknowledgments}

None.

\section{Conflict of interest}

The authors declare that there is no conflict of interest.

\section{References}

1. Rodini CO, Lopes NM, Lara VS, et al. Oral cancer stem cells - Properties and consequences. J Appl Oral Sci. 2017;25(6):708-715.

2. Chi AC, Day TA, Neville BW. Oral cavity and oropharyngeal squamous cell carcinoma-an update. CA Cancer J Clin. 2015;65(5):401-421.
3. Malik UU, Zarina S, Pennington SR. Oral squamous cell carcinoma: Key clinical questions, biomarker discovery, and the role of proteomics. Arch Oral Biol. 2016;63:53-65.

4. Yu QR. Stem cells and cancer stem cells. Journal of Clinical Rehabilitative Tissue Engineering Research.

5. Buczacki S. Cancer Stem Cells. Encyclopedia of Cell Biology. 2015.

6. Li L, Neaves WB. Normal stem cells and cancer stem cells: The niche matters. Cancer Research. 2006.

7. Dalerba P, Cho RW, Clarke MF. Cancer Stem Cells: Models and Concepts. Annu Rev Med. 2007;58:267-284.

8. Bonnet D, Dick JE. Human acute myeloid leukemia is organized as a hierarchy that originates from a primitive hematopoietic cell. Nat Med. 1997;3(7):730-737.

9. Dick JE, Bonnet D. Human Acute Myeloid Leukaemia is organised as a heirarchy that originates from the primitive haematopoetic cell. Nat Med. 1997;3:730-737.

10. Colombatti A, Danussi C, Pivetta E, et al. Cancer stem cells and the microenvironment. 2012.

11. Clarke MF, Dick JE, Dirks PB, et al. Cancer stem cells - Perspectives on current status and future directions: AACR workshop on cancer stem cells. Cancer Res. 2006;66(19):9339-9344.

12. Nguyen LV, Vanner R, Dirks P, et al. Cancer stem cells: An evolving concept. Nat Rev Cancer. 2012;12(12):133-143.

13. Batlle E, Clevers H. Cancer stem cells revisited. Nat Med. 2017.

14. Clarke MF, Fuller M. Stem Cells and Cancer: Two Faces of Eve. Cell. 2006;124(6):1111-1115.

15. McCulloch EA. Stem cells in normal and leukemic hemopoiesis. Blood. 1983;62(1):1-13.

16. Lapidot T, Kollet O. The essential roles of the chemokine SDF-1 and its receptor CXCR4 in human stem cell homing and repopulation of transplanted immune-deficient NOD/SCID and NOD/SCID/B2mnull mice. Leukemia. 2002;16(10):1992-2003.

17. Mehrotra B, George TI, Kavanau K, et al. Cytogenetically aberrant cells in the stem cell compartment (CD34+lin-) in acute myeloid leukemia. Blood. 1995;86(3):1139-1147.

18. Taussig DC, Miraki-Moud F, Anjos-Afonso F, et al. Anti-CD38 antibody Mediated clearance of human repopulating cells masks the heterogeneity of leukemia-initiating cells. Blood. 2008;112(3):568-575.

19. Yan Y, Zuo X, Wei D. Concise Review: Emerging Role of CD44 in Cancer Stem Cells: A Promising Biomarker and Therapeutic Target. Stem Cells Transl Med. 2015;4(9):1033-1043.

20. Zou B, Sun S, Qi X, et al. Aldehyde dehydrogenase activity is a cancer stem cell marker of tongue squamous cell carcinoma. Mol Med Rep. 2012;5(4):1116-1120.

21. Poage GM, Christensen BC, Houseman EA, et al. Genetic and epigenetic somatic alterations in head and neck squamous cell carcinomas are globally coordinated but not locally targeted. PLoS One. 2010;5(3):e9651.

22. Barker N, Tan S, Clevers H. Lgr proteins in epithelial stem cell biology. Development. 2013;140(12):2484-2494. 